\title{
On a Sufficient Condition for Well-posedness in Gevrey Classes of Some Weakly Hyperbolic Cauchy Problems
}

\author{
By \\ Shigeharu IтоH*
}

\section{Introduction}

In this paper we shall study well-posedness of the Cauchy problem for some weakly hyperbolic operators in Gevrey classes. That is to say, we consider whether we can determine a function space in which the Cauchy problem for given weakly hyperbolic operator is well-posed or not.

This question has been studied by several mathematicians.

The results independent of the lower order terms were obtained by Ohya [8], Leray-Ohya [6], Steinberg [9], Ivrii [3], Trepreau [10], Bronstein [1], Kajitani [5] and Nishitani [7], which show that the multiplicity of the characteristic roots determines the well-posed class.

On the other hand, in [4] Ivrii presented two interesting examples.

(I) Let $P=\partial_{t}^{2}-t^{2 \mu} \partial_{x}^{2}+a t^{\nu} \partial_{x}$, where $\mu, \nu$ are non-negative integers and $a$ is a non-zero constant. When $0 \leqq \nu<\mu-1$, the Cauchy problem for $P$ is $\gamma_{i o c}^{(\kappa)}$-wellposed, if and only if $1 \leqq \kappa<(2 \mu-\nu) /(\mu-\nu-1)$.

(II) Let $P=\partial_{t}^{2}-x^{2} \iota_{x}^{2}+a x^{\nu} \partial_{x}$, where $\mu, \nu$ are non-negative integers and $a$ is a non-zero constant. When $0 \leqq \nu<\mu$, the Cauchy problem for $P$ is $\gamma_{l o c}^{(\pi)}$-wellposed, if and only if $1 \leqq \kappa<(2 \mu-\nu) /(\mu-\nu)$. These two cases show that the lower order terms have a great effect on the well-posed class.

Igari [2] and Uryu [12] extended ( I ) for more general operators respectively and Uryu-Itoh [13] extended (II) for second order weakly hyperbolic operators.

In this article we shall consider the most general case of (II).

\section{$\S 1$. Statement of the Result and Remarks}

Let $(x, t) \in \boldsymbol{R}^{n} \times[0, T]$ and $\left(D_{x}, D_{t}\right)=\left(D_{x_{1}}, \cdots, D_{x_{n}}, D_{t}\right)=\left(-\sqrt{-1} \partial_{x_{1}}, \cdots\right.$, $\left.-\sqrt{-1} \partial_{x_{n}},-\sqrt{-1} \partial_{t}\right)$. Let us denote by $(\xi, \tau)$ the dual variable of $(x, t)$.

Communicated by S. Matsuura, February 16, 1985.

* Department of Mathematics, School of Science and Engineering. Waseda University, Tokyo 160, Japan. 
Next we shall define the Gevrey classes.

Definition 1.1. $\left(\gamma_{i o c}^{(\kappa)}, \gamma^{(\kappa)} ; \kappa>1\right) f(x) \in \gamma_{\text {ioc }}^{(n)}$ implies that $f(x) \in C^{\infty}\left(\boldsymbol{R}^{n}\right)$ and for any compact set $K \subset \boldsymbol{R}^{n}$ there exist constants $c, R>0$ such that

$$
\left|D_{x}^{\alpha} f(x) ! \leqq c R^{|\alpha|}\right| \alpha ! !^{\kappa}, x \in K, \quad \text { for any } \alpha .
$$

$f(x) \in \gamma^{(\kappa)}$ implies that $f(x) \in C^{\infty}\left(\boldsymbol{R}^{n}\right)$ and (1.1) holds for any $x \in \boldsymbol{R}^{n}$.

Let $L=L\left(x, t, D_{x}, D_{t}\right)=L_{0}\left(x, t, D_{x}, D_{t}\right)+L_{1}\left(x, t, D_{x}, D_{t}\right)$, where

$$
L_{0}\left(x, t, D_{x}, D_{t}\right)=D_{t}^{m}+\sum_{\substack{|\alpha| \jmath=m \\ j \leq m-1}} \sigma(x)^{|\alpha| \mu} a_{\alpha, \jmath}(x, t) D_{x}^{\alpha} D_{t}^{j}
$$

and

$$
L_{1}\left(x, t, D_{x}, D_{i}\right)=\sum_{|\alpha|+\jmath \leqq m-1} \sigma(x)^{\nu \alpha, j} a_{\alpha, \jmath}(x, t) D_{x}^{\alpha} D_{t}^{j} .
$$

We assume the following conditions on $L$.

(1.4) $\tau$-roots of

$$
\widetilde{L}_{0}(x, t, \xi, \tau)=\tau^{m}+\sum_{\substack{\mid \alpha+j=m \\ j \leq m-1}} a_{\alpha, j}(x, t) \xi^{\alpha} \tau^{j}=0
$$

are real and distinct.

$$
a_{\alpha, j}(x, t) \in \mathscr{B}\left([0, T], \gamma^{(\kappa)}\right) .
$$

(1.6) $\sigma(x) \in \gamma^{(\kappa)}$ and is a real-valued function.

(1.7) $\mu$ is a positive integer and $\nu_{\alpha, j}$ are non-negative integers.

Now we shall define $\rho$ as follows.

$$
\rho=\max _{0 \leqq|\alpha|+j \leqq m-1}\left\{\left(m-j-\nu_{\alpha, j} / \mu\right) /(m-j-|\alpha|), 1\right\} .
$$

Then we have

Theorem 1.1. Under (1.4)-(1.7), if $1 \leqq \kappa<\rho /(\rho-1)$, the Cauchy problem for $L$ :

$$
\left\{\begin{array}{l}
L u(x, t)=f(x, t) \quad \text { in } \quad \mathbb{R}^{n} \times(0, T] \\
\left.D_{t}^{i} u(x, t)\right|_{t=0}=u^{i}(x), i=0, \cdots, m-1 \quad \text { on } \quad \mathbb{R}^{n}
\end{array}\right.
$$

is $\gamma_{\text {loc }}^{(\kappa)}$-well-posed, $i$. e. for any $u^{i}(x) \in \gamma_{i o c}^{(\kappa)}(i=0, \cdots, m-1)$ and any $f(x, t) \in$ $\mathscr{B}\left([0, T], \gamma_{l o c}^{(k)}\right)$, there exists a unique solution $u(x, t) \in \mathscr{B}\left([0, T], \gamma_{l o c}^{(\kappa)}\right)$ of $(1.9)$.

Remark 1.1. When $\rho=1,(1.9)$ is $C^{\infty}$-well-posed.

Remark 1.2. In the case of the finite degeneracy our sufficient condition is best.

Remark 1.3. From Remark 1.1, we may only consider the case that $0 \leqq \nu_{\alpha, j}$ $\leqq|\alpha| \mu$. 


\section{§. Proof of Theorem 1.1}

In this section we shall reduce Theorem 1.1 to Theorem 2.1.

Definition 2.1. We say that $f(x) \in H^{\infty}$ belongs to $\Gamma^{(\kappa)}$ if there exist constants $c, R>0$ such that

$$
\left\|D_{x}^{\alpha} f(x)\right\| \leqq c R^{|\alpha|}|\alpha| !^{\kappa} \quad \text { for any } \alpha,
$$

where $\|\cdot\|$ denotes $L^{2}$-norm with respect to $x$.

Let $P=P\left(x, t, D_{x}, D_{t}\right)=P_{0}\left(x, t, D_{x}, D_{t}\right)+P_{1}\left(x, t, D_{x}, D_{t}\right)$.

$$
P_{0}(x, t, \xi, \tau)=\prod_{\jmath=1}^{m}\left(\tau-\sigma(x)^{\mu} \lambda_{j}(x, t, \xi)\right),
$$

where $\lambda_{\jmath}(x, t, \xi) \in \mathscr{B}\left([0, T], S^{1}(\kappa)\right)$ are real-valued and $\left|\left(\lambda_{i}-\lambda_{\jmath}\right)(x, t, \xi)\right| \geqq \delta\langle\xi\rangle$ for some constant $\delta>0$ if $i \neq j$. Further

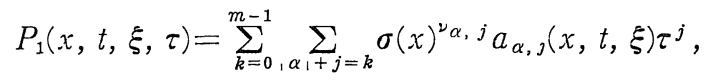

where $a_{a, j}(x, t, \xi) \in \mathscr{B}\left([0, T], S^{|\alpha|}(\kappa)\right)$. Here $S^{j}(\kappa)$ are symbol classes defined in Appendix.

Then we get the following theorem.

Theorem 2.1. Under (1.4)-(1.7), if $1 \leqq \kappa<\rho /(\rho-1)$, the Cauchy problem for $P$ is $\Gamma^{(\kappa)}$-well-posed.

In order to prove Theorem 1.1, it is sufficient to show Theorem 2.1. For since an operator $L$ is changed into above operator $P$ by spacelike transformation, we can see that a domain of dependence is finite. Hence using a partition of unity Theorem 1.1 follows from Theorem 2.1.

We shall prove Theorem 2.1 by the method of successive approximations. Therefore we decompose $P$ as follows and consider the following scheme.

$$
P\left(x, t, D_{x}, D_{t}\right)=Q_{0}\left(x, t, D_{x}, D_{t}\right)+Q_{1}\left(x, t, D_{x}, D_{t}\right),
$$

where as $\nu_{\alpha, \jmath}=|\alpha| \mu$

$$
Q_{0}\left(x, t, D_{x}, D_{t}\right)=P_{0}\left(x, t, D_{x}, D_{t}\right)+\sum_{k=0|\alpha|+\jmath=k}^{m-1} \sum_{i} \sigma(x)^{\nu \alpha, j} a_{\alpha, \jmath}\left(x, t, D_{x}\right) D_{t}^{j}
$$

and as $0 \leqq \nu_{o, j}<|\alpha| \mu$

$$
\begin{aligned}
& Q_{1}\left(x, t, D_{x}, D_{t}\right)=\sum_{k=1}^{m-1} \sum_{\substack{|\alpha|+>=k,|a| \neq=0}} \sigma(x)^{\nu} a, j a_{c, j}\left(x, t, D_{x}\right) D_{t}^{j} . \\
& \left\{\begin{array}{l}
Q_{0} u_{0}(x, t)=f(x, t) \quad \text { in } \mathbb{R}^{n} \times(0, T] \\
\left.D_{t}^{i} u_{0}(x, t)\right|_{t=0}=u^{i}(x), 0 \leqq i \leqq m-1 \quad \text { on } \mathbb{R}^{n}
\end{array}\right.
\end{aligned}
$$


and for $j \geqq 1$

$$
\begin{cases}Q_{0} u_{\jmath}(x, t)=-Q_{1} u_{\jmath-1}(x, t) \quad \text { in } & \boldsymbol{R}^{n} \times(0, T] \\ \left.D_{t}^{\imath} u_{\jmath}(x, t)\right|_{t=0}=0,0 \leqq i \leqq m-1 & \text { on } \quad \boldsymbol{R}^{n}\end{cases}
$$

Here we refer to Uryu [11].

Proposition 2.1. The Cauchy problem for $Q_{0}$ is $H^{\infty}$-well-posed.

Since $\Gamma^{(\kappa)} \subset H^{\infty}, u_{0}(x, t)$ which is a solution of $(2.6)_{0}$ belongs to $\mathscr{B}([0, T]$, $H^{\infty}\left(\boldsymbol{R}^{n}\right)$ ) by Proposition 2.1. If we note that $Q_{1}$ is a pseudo-differential operator in $x$, then we obtain that $Q_{1} u_{0} \in \mathscr{B}\left([0, T], H^{\infty}\left(\boldsymbol{R}^{n}\right)\right)$. Hence it follows from $(2.6)_{1}$ and Proposition 2.1 that $u_{1}(x, t) \in \mathscr{B}\left([0, T], H^{\infty}\left(\boldsymbol{R}^{n}\right)\right)$. Repeating these steps we get that for any $j \geqq 0, u_{\jmath}(x, t) \in \mathscr{B}\left([0, T], H^{\infty}\left(\boldsymbol{R}^{n}\right)\right)$. Therefore it is sufficient to show that the formal solution

$$
u(x, t)=\sum_{j=0}^{\infty} u_{\jmath}(x, t)
$$

converges in $\mathscr{B}\left([0, T], \Gamma^{(\kappa)}\right)$.

Our plan is as follows. In $\S 3$ we shall get an energy inequality for $Q_{0}$ in $L^{2}$. In $\S 4$ we shall estimate derivatives of a solution of $(2.8)$ :

$$
\left\{\begin{array}{l}
Q_{0} v(x, t)=g(x, t) \\
\left.D_{t}^{i} v(x, t)\right|_{t=0}=0, \quad 0 \leqq i \leqq m-1
\end{array}\right.
$$

where $g(x, t) \in \mathscr{B}\left([0, T], \Gamma^{(\kappa)}\right)$ such that for any fixed integer $s \geqq\left. 1 D_{t}^{i} g(x, t)\right|_{t=0}$ $=0,0 \leqq i \leqq s-1$. And in $\S 5$ we shall obtain estimates of $Q_{1} v(x, t)$. Using the consequence in $\S 4$ and $\S 5$, we shall prove Theorem 2.1 in $\S 6$.

\section{$\S 3$. Energy Inequality for $Q_{0}$}

The aim of this section is to show the following lemma.

Lemma 3.1. Let $\Phi(t)=\sum_{k=0}^{m-1} a^{m-(k+1)} \sum_{i+\jmath=k}\left\|\sigma(x)^{i \mu} \Lambda^{i} D_{t}^{j} u\right\|$, where $\Lambda$ is the pseudo-differential operator with symbol $\langle\xi\rangle=\left(1+|\xi|^{2}\right)^{1 / 2}$ and $a \geqq 1$. Then there exists a constant $c^{\prime}>0$ such that

$$
\Phi(t) \leqq c^{\prime} \int_{0}^{t}\left\{\left\|Q_{0} u\right\|+a \Phi(\tau)\right\} d \tau
$$

for $u(x, t) \in \mathscr{B}\left([0, T], H^{\infty}\left(\boldsymbol{R}^{n}\right)\right),\left.D_{t}^{i} u\right|_{t=0}=0,0 \leqq i \leqq m-1$.

In order to prove Lemma 3.1 we prepare several lemmas.

Let $\partial_{\jmath}=D_{t}-\sigma(x)^{\mu} \lambda_{\jmath}\left(x, t, D_{x}\right), 1 \leqq j \leqq m$. We note that $\lambda_{\jmath} \in \mathscr{B}\left([0, T], S^{1}\right)$ and there exists a constant $\delta>0$ such that $\left|\left(\lambda_{2}-\lambda_{j}\right)(x, t, \xi)\right| \geqq \delta\langle\xi\rangle$ if $i \neq j$.

Lemma 3.2. For $i, j$ with $1 \leqq i, j \leqq m$, there exist pseudo-differential operators 
$A_{i \jmath}, B_{\imath \jmath}$ and $C_{\imath \jmath} \in \mathscr{B}\left([0, T], S^{0}\right)$ such that

$$
\left[\partial_{\imath}, \partial_{\jmath}\right]=A_{\imath \jmath} \partial_{\imath}+B_{\imath \jmath} \partial_{\jmath}+C_{\imath \jmath},
$$

where $[\cdot, \cdot]$ is the commutator.

Proof. Let $\sigma_{0}\left(\left[\partial_{\imath}, \partial_{j}\right]\right)$ be the principal symbol of $\left[\partial_{\imath}, \partial_{j}\right]$. Then by the product formula of pseudo-differential operators, we get

$$
\begin{aligned}
\sigma_{0}\left(\left[\partial_{\imath}, \partial_{\jmath}\right]\right)= & \sum_{k=0}^{n}\left\{\partial_{\xi_{k}}\left(\xi_{0}-\sigma(x)^{\mu} \lambda_{2}\right) D_{x_{k}}\left(\xi_{0}-\sigma(x)^{\prime \prime} \lambda_{\jmath}\right)\right. \\
& \left.-\partial_{\xi_{k}}\left(\xi_{0}-\sigma(x)^{\prime \prime} \lambda_{\jmath}\right) D_{x_{k}}\left(\xi_{0}-\sigma(x)^{\prime \prime} \lambda_{\imath}\right)\right\} \\
= & \sigma(x)^{\prime \prime} D_{\imath}(x, t, \xi),
\end{aligned}
$$

where $D_{\imath \jmath} \in \mathscr{B}\left([0, T], S^{1}\right)$. Here we use the notation $x_{0}=t, \xi_{0}=\tau$.

If we set $A_{i \jmath}=D_{i \jmath} /\left(\lambda_{\jmath}-\lambda_{i}\right)$ and $B_{\imath \jmath}=D_{i \jmath} /\left(\lambda_{i}-\lambda_{\jmath}\right)$, then $A_{\imath \jmath}, B_{\imath \jmath} \in \mathscr{B}\left([0, T], S^{0}\right)$ and $A_{\imath \jmath}\left(\xi_{0}-\sigma(x)^{\prime \prime} \lambda_{\imath}\right)+B_{i \jmath}\left(\xi_{0}-\sigma(x)^{\mu} \lambda_{\jmath}\right)=\sigma(x)^{\mu} D_{\imath \jmath}$.

Now we consider the modules $W_{k}(0 \leqq k \leqq m-1)$ over the ring of pseudodifferential operators in $x$ of order zero.

Let $\Pi_{m}=\partial_{1} \partial_{2} \cdots \partial_{m}$. Let $W_{m-1}$ be the module generated by the monomial operators $\Pi_{m} / \partial_{i}=\partial_{1} \cdots \check{\partial}_{i} \cdots \partial_{m}$ of order $m-1$ and let $W_{m-2}$ be the module generated by the operators $\Pi_{m} / \partial_{i} \partial_{j}(i \neq j)$ of order $m-2$ and so on.

Lemma 3.3. For any monomial $\omega_{k}^{\alpha} \in W_{k}(0 \leqq k \leqq m-1)$, there exist $\partial_{\imath}$ and $\omega_{k+1}^{\beta} \in W_{k+1}$ such that

$$
\partial_{\imath} \omega_{k}^{\alpha}=\omega_{k+1}^{\beta}+\sum_{\jmath=1}^{k+1} \sum_{\gamma} C_{\gamma, \jmath} \omega_{k+1-\jmath}^{\gamma},
$$

where $C_{r j} \in \mathscr{B}\left([0, T], S^{0}\right)$.

Proof. For any $\omega_{k}^{\alpha}=\partial_{j_{1}} \cdots \partial_{\jmath_{k}}\left(j_{1}<\cdots<j_{k}\right)$, there exists some $j \notin\left\{i_{1}, \cdots, j_{k}\right\}$ with $1 \leqq j \leqq m$. Hence if we use Lemma 3.2 , we easily obtain (3.3). Q.E.D.

Lemma 3.4. Let $\Psi(t)$ be

$$
\Psi(t)=\sum_{k=0}^{m-1} \sum_{a} a^{m-(k+1)}\left\|\omega_{k}^{a} u\right\|
$$

for $u(x, t) \in \mathscr{B}\left([0, T], H^{\infty}\right)$ and $a \geqq 1$. Then we have the following energy inequality

$$
\frac{d}{d t} \Psi(t) \leqq c_{1} a \Psi(t)+\left\|\Pi_{m} u\right\|
$$

Proof. By Lemma 3.3

$$
\partial_{\imath} \omega_{k}^{a} u=\omega_{k+1}^{\beta} u+\sum_{\jmath=1}^{k+1} \sum_{\gamma} C_{i \jmath} \omega_{k+1-\jmath}^{\gamma} u .
$$


If we set $v=\omega_{k}^{\alpha} u$ and $g=\omega_{k+1}^{3} u+\sum_{j=1}^{k+1} \sum_{\gamma} C_{\gamma j} \omega_{k+1-j}^{\gamma} u$, then

Hence we get

$$
\begin{aligned}
\frac{d}{d t}\|v\|^{2} & =2 \operatorname{Re}\left(\frac{d}{d t} v, v\right) \\
& =2 \operatorname{Re}\left(\sqrt{-1} g+\sqrt{-1} \sigma(x)^{\mu} \lambda_{i} v, v\right) \\
& \leqq 2\|g\|\|v\|+c_{2}\|v\|^{2} .
\end{aligned}
$$

$$
\frac{d}{d t}\|v\| \leqq\left\|\omega_{k+1}^{\beta} u\right\|+c_{3}\left\{\left\|\omega_{k}^{\alpha} u\right\|+\sum_{\jmath=1}^{k+1} \sum_{\gamma}\left\|\omega_{k+1-\jmath}^{\gamma} u\right\|\right\} .
$$

For any $k$ with $0 \leqq k \leqq m-2$, we have

$$
\begin{aligned}
& a^{m-(k+1)} \frac{d}{d t}\left\|\omega_{k}^{\alpha} u\right\| \\
& \quad \leqq a^{m-(k+1)}\left\|\omega_{k+1}^{\beta} u\right\|+c_{3}\left\{a^{m-(k+1)}\left\|\omega_{k}^{\alpha} u\right\|+\sum_{j=1}^{k+1} \sum_{\gamma} a^{m-(k+1)}\left\|\omega_{k+1-j}^{\gamma} u\right\|\right\} \\
& \quad \leqq a a^{m-(k+2)}\left\|\omega_{k+1}^{\beta} u\right\|+c_{3}\left\{a^{m-(k+1)}\left\|\omega_{k}^{\alpha} u\right\|+\sum_{j=1}^{k+1} \sum_{\gamma} a^{m-(k-j+2)}\left\|\omega_{k+1-j}^{\gamma} u\right\|\right\} \\
& \quad \leqq a \Psi(t)+c_{4} \Psi(t) \\
& \quad \leqq c_{5} a \Psi(t) .
\end{aligned}
$$

Similarly when $k=m-1$, we obtain

$$
\frac{d}{d t}\left\|\omega_{m-1}^{\alpha} u\right\| \leqq\left\|\Pi_{m} u\right\|+c_{6} \Psi(t) . \quad \text { Q. E. D. }
$$

Lemma 3.5. Let $\Pi_{s}=\partial_{i_{1}} \cdots \partial_{i_{s}}\left(1 \leqq i_{1}<\cdots<i_{s} \leqq m\right)$. Then $\sigma\left(\Pi_{s}\right)$, the symbol of $\Pi_{s}$, is expressed in the form

$$
\sigma\left(\Pi_{s}\right)=\prod_{\jmath=1}^{s}\left(\tau-\sigma(x)^{\mu} \lambda_{i_{j}}\right)+R_{s-1}+\cdots+R_{0},
$$

where $R_{s-j}=\sum_{p+q=s-j} \sigma(x)^{p \mu} b_{p j}(x, t, \xi) \tau^{q}$ for some $b_{p j} \in \mathscr{B}\left([0, T], S^{p}\right), j=1, \cdots, s$.

Proof. We carry out the proof by induction on $s$. When $s=1,(3.6)$ is trivial. Suppose (3.6) holds for $s$. Since $\Pi_{s+1}=\Pi_{s} \partial_{i_{s+1}}$,

$$
\sigma\left(\Pi_{s+1}\right)=\sigma\left(\Pi_{s}\right)\left(\xi_{0}-\sigma(x)^{\mu} \lambda_{i_{s+1}}\right)+\sum_{\alpha \neq 0} \partial_{\xi}^{\alpha} \sigma\left(\Pi_{s}\right) D_{x}^{\alpha}\left(\xi_{0}-\sigma(x)^{\mu} \lambda_{i_{s+1}}\right) .
$$

Substituting the right hand side of (3.6) for $\sigma\left(\Pi_{s}\right)$, we have (3.6) with $s+1$.

Q.E.D.

Corollary 3.1. There exist pseudo-differential operators $C_{i j}\left(x, t, D_{x}\right) \in$ $\mathscr{B}\left([0, T], S^{i}\right)$ such that

$$
Q_{0}-\Pi_{m}=\sum_{k=0}^{m-1} \sum_{i+\jmath=k} \sigma(x)^{i \mu} C_{i \jmath}\left(x, t, D_{x}\right) D_{t}^{j} .
$$


Proof. From Lemma 3.5 with $s=m$ and the form of $Q_{0},(3.7)$ is verified.

Q.E.D.

Lemma 3.6. There exists a constant $c_{7}>0$ such that

$$
c_{7}^{-1} \Phi(t) \leqq \Psi(t) \leqq c_{7} \Phi(t) .
$$

Proof. In order to see that $\Phi(t) \leqq c_{8} \Psi(t)$, it is sufficient to show the following. There exist $A,(x, t, \xi) \in \mathscr{B}\left([0, T], S^{0}\right)$ such that for $i^{\prime}+j^{\prime}=m-k, 1 \leqq k \leqq m$,

$$
\sigma(x)^{i^{\prime} \mu}\langle\xi\rangle^{i^{\prime}} \tau^{j^{\prime}}=\sum_{j=k}^{m} A_{j}(x, t, \xi) \prod_{\substack{i \neq j \\ i \geq k}}\left(\tau-\sigma(x)^{\mu} \lambda_{\imath}\right) .
$$

Substituting $\sigma(x)^{\mu} \lambda$, for $\tau$, then we obtain

$$
a_{\jmath}(x, t, \xi)\langle\xi\rangle^{m-k}=A_{\jmath}(x, t, \xi) \prod_{\substack{i \neq j \\ i \geq k}}\left(\lambda_{\jmath}-\lambda_{i}\right), \quad \text { where } a_{\jmath} \in \mathscr{B}\left([0, T], S^{0}\right) .
$$

Therefore we set

$$
A_{\jmath}(x, t, \xi)=a,\langle\xi\rangle^{m-k}\left\{\prod_{\substack{i \neq j \\ i \geq k}}\left(\lambda_{j}-\lambda_{i}\right)\right\}^{-1} .
$$

On the other hand, it is trivial to see that $\Psi(t) \leqq c_{9} \Phi(t)$.

Q.E.D.

Proof of Lemma 3.1. By Corollary 3.1 and Lemma 3.6, we have

$$
\begin{aligned}
\left\|\Pi_{m} u\right\| & \leqq\left\|\left(Q_{0}-\Pi_{m}\right) u\right\|+\left\|Q_{0} u\right\| \leqq c_{10} \Phi(t)+\left\|Q_{0} u\right\| \\
& \leqq c_{7} c_{10} \Psi(t)+\left\|Q_{0} u\right\| .
\end{aligned}
$$

And from Lemma 3.4,

$$
\Psi(t) \leqq c_{11} \int_{0}^{t}\left\{\left\|Q_{0} u\right\|+a \Psi(\tau)\right\} d \tau
$$

Using Lemma 3.6 again, we can obtain

$$
\Phi(t) \leqq c^{\prime} \int_{0}^{t}\left\{\left\|Q_{0} u\right\|+a \Phi(\tau)\right\} d \tau
$$

This completes the proof.

Q.E.D.

\section{$\S 4$. Estimate of $\Lambda^{r} v$}

We assume the existence of solutions of the following Cauchy problem:

$$
\left\{\begin{array}{l}
Q_{0} v(x, t)=g(x, t) \\
\left.D_{t}^{i} v(x, t)\right|_{t=0}=0, \quad 0 \leqq i \leqq m-1
\end{array}\right.
$$

where $g(x, t) \in \mathscr{B}\left([0, T], \Gamma^{(\kappa)}\right)$ such that for any fixed integer $s \geqq\left. 1 D_{\imath}^{2} g(x, t)\right|_{t=0}$ $=0,0 \leqq i \leqq s-1$.

Therefore we may assume that for any $r \geqq 0$ there exist constants $c, R$, $M>0$ such that

$$
\left\|\Lambda^{r} g(x, t)\right\| \leqq c R^{r} \gamma !{ }^{k} t^{s} e^{1 I r t}
$$


For simplicity we use the notation

$$
w_{r}(s, t, R)=R^{r} r !{ }^{\kappa} t^{s} e^{M r t} .
$$

We shall prove the basic lemma of this section.

Lemma 4.1. Let $\Phi_{r}(t)$ be

$$
\Phi_{r}(t)=\sum_{k=0}^{m-1}(r+1)^{m-(k+1)} \sum_{i+\jmath=k}\left\|\sigma(x)^{\imath \mu} \Lambda^{r+i} D_{t}^{j} v\right\| .
$$

Then for any $r \geqq 0$ there exists a constant $A>0$ such that for sufficiently large $R, M, s$

$$
\Phi_{r}(t) \leqq c 4 s^{-1} w_{r}(s, t, R) .
$$

Proof. We carry out the proof by induction on $r$.

When $r=0$, it follows from Lemma 3.1 and (4.1) that

$$
\Phi_{0}(t) \leqq c^{\prime} \int_{0}^{t} c w_{0}(s, \tau, R) d \tau+c^{\prime} \int_{0}^{t} \Phi_{0}(\tau) d \tau
$$

By Gronwall's inequality, we get

$$
\Phi_{0}(t) \leqq c A s^{-1} w_{0}(s, t, R)
$$

if we choose $A$ such that $A \geqq c^{\prime} T e^{c^{\prime} T}$.

We assume that (4.2) is valid for any $r$ such that $0 \leqq r \leqq n$.

Let us show that (4.2) is valid for $r=n+1$. For $r>0$, operating the pseudodifferential operator $\Lambda^{r}$ on both sides of $Q_{0} v=g$, we get

$$
Q_{0} \Lambda^{r} v=\Lambda^{r} g+\left[Q_{0}, \Lambda^{r}\right] v .
$$

We shall estimate the commutator $\left[Q_{0}, \Lambda^{r}\right] v$. We note that

$$
Q_{0}(x, t, \xi, \tau)=\tau^{m}+\sum_{\substack{i+j \leq m \\ j \leq m-1}} \sigma(x)^{i \mu} a_{\imath}(x, t, \xi) \tau^{j},
$$

where $a_{\imath} \in \mathscr{B}\left([0, T], S^{i}(\kappa)\right)$. Therefore we have

$$
\left[Q_{0}, \Lambda^{r}\right]=\sum_{\substack{i+j \leq m \\ j \leq m-1}}\left[\sigma(x)^{i \mu} a_{i}, \Lambda^{r}\right] D_{t}^{j} .
$$

By the fomula of pseudo-differential operator, we obtain

$$
\sigma\left(\left[\sigma(x)^{i \mu} a_{\imath}, \Lambda^{r}\right]\right)=\sum_{k=1}^{r+i-1} \sum_{|a|=k} \frac{1}{\alpha !} \partial_{\xi}^{\alpha}\langle\xi\rangle^{r} D_{x}^{\alpha}\left\{\sigma(x)^{i \mu} a_{\imath}\right\}+r_{\imath}(x, t, \xi) .
$$

It follows from Lemma A. 3 in Appendix and $\mu \geqq 1$ that

$$
\begin{aligned}
& \left\|\left[\sigma(x)^{i \mu} a_{\imath}, \Lambda^{r}\right] D^{j} v\right\| \\
& \leqq \\
& \quad \hat{c} r\left\|\sigma(x)^{i \mu-1} \Lambda^{r+i-1} D_{t}^{j} v\right\|+\cdots+\hat{c} r^{i}\left\|\sigma(x)^{i \mu-i} \Lambda^{r} D^{j} v\right\| \\
& \quad+\sum_{k=\imath+1}^{r} \hat{c} \hat{R}^{k-i}(k-i) !{ }^{\kappa}\left(\begin{array}{c}
r \\
k
\end{array}\right)\left\|\Lambda^{r+i-k} D^{j} v\right\|
\end{aligned}
$$




$$
\begin{aligned}
& +\sum_{k=r+1}^{r+i-1} \hat{c} \hat{R}^{k-\imath}(k-i) !{ }^{k}\left\|\Lambda^{r+\imath-k} D_{t}^{\jmath} v\right\| \\
& +\hat{c} \hat{R}^{r} r !^{\kappa}\left\|D_{t}^{j} v\right\| \\
& \leqq c_{12} r\left\{\left\|\sigma(x)^{\mu^{(i-1)}} \Lambda^{r+2-1} D_{t}^{j} v\right\|+\cdots+r^{2-1}\left\|A^{r} D_{t}^{j} v\right\|\right\} \\
& +\sum_{k=i+1}^{r}\left(\frac{\hat{R}}{R}\right)^{k-i}\left(\begin{array}{l}
r \\
k
\end{array}\right)\left(\begin{array}{c}
r \\
k-i
\end{array}\right)^{-\kappa}(r+i-k+1)^{-(m-j-1)} c^{-1} s^{-1} w,(s, t, R) \\
& +\sum_{k=r+1}^{r+i-1}\left(\frac{\hat{R}}{R}\right)^{k-i}\left(\begin{array}{c}
r \\
k-i
\end{array}\right)^{-k} c A s^{-1} w_{r}(s, t, R) \\
& +\left(\frac{\hat{R}}{R}\right)^{r} c A s^{-1} w_{r}(s, t, R) \text {. }
\end{aligned}
$$

Hence we make $R \geqq 2 \hat{R}$, and get

$$
\begin{aligned}
\left\|\left[Q_{0}, A^{r}\right] v\right\| & \leqq c_{13}\left\{r \Phi_{r}(t)+r c A s^{-1} w_{r}(s, t, R)+c \cdot s^{-1} w_{r}(s, t, R)\right\} \\
& \leqq c_{14}\left\{r \Phi_{r}(t)+r c A s^{-1} w_{r}(s, t, R)\right\} .
\end{aligned}
$$

We note that $c_{14}$ is independent of $r$.

From Lemma 3.1,

$$
\begin{aligned}
\Phi_{r}(t) & \leqq c^{\prime} \int_{0}^{t}\left\{\left\|\Lambda^{r} g\right\|+\left\|\left[Q_{0}, \Lambda^{r}\right] v\right\|+(r+1) \Phi_{r}(\tau)\right\} d \tau \\
& \leqq c^{\prime} \int_{0}^{t}\left\{c w_{r}(s, \tau, R)+c_{15}(r+1) \Phi_{r}(\tau)+c_{14} r c A s^{-1} w_{r}(s, \tau, R)\right\} d \tau .
\end{aligned}
$$

Let $f(t)=c^{\prime} \int_{0}^{t}\left\{c w_{r}(s, \tau, R)+c_{14} r c A s^{-1} w_{r}(s, \tau, R)\right\} d \tau$, then

$$
\Phi_{r}(t) \leqq f(t)+c_{16} r \int_{0}^{t} \Phi_{r}(\tau) d \tau
$$

Therefore we obtain

$$
\Phi_{r}(t) \leqq f(t) \mid-c_{16} r \int_{0}^{t} f(\tau) e^{c_{16} r(t-\tau)} d \tau
$$

Now we calculate $f(t)$.

$$
\begin{aligned}
f(t) & =c^{\prime} c \int_{0}^{t} R^{r} r !{ }^{k} \tau^{s} e^{M I r \tau} d \tau+r c^{\prime} c_{14} c A s^{-1} \int_{0}^{t} R^{r} r !{ }^{k} \tau^{s} e^{I I r \tau} d \tau \\
& \leqq c^{\prime} c T s^{-1} w_{r}(s, t, R)+c^{\prime} c_{14} c A s^{-1} M^{-1} w_{r}(s, t, R) \\
& \leqq(c A / 2) s^{-1} w_{r}(s, t, R), \text { if we make } A \geqq 4 c^{\prime} T \text { and } M \geqq 4 c^{\prime} c_{14} .
\end{aligned}
$$

Hence we get

$$
\begin{aligned}
\Phi_{r}(t) & \leqq c A 2^{-1} s^{-1} w_{r}(s, t, R)+c_{16} r \int_{0}^{t} c A 2^{-1} s^{-1} w_{r}(s, \tau, R) e^{c_{16} r(t-\tau)} d \tau \\
& \leqq c A 2^{-1} s^{-1} w_{r}(s, t, R)+c A 2^{-1} s^{-1} c_{16}\left(M-c_{16}\right)^{-1} w_{r}(s, t, R) \\
& \leqq c A s^{-1} w_{r}(s, t, R),
\end{aligned}
$$


where $M$ is a sufficiently large number such that $c_{16}\left(M-c_{16}\right)^{-1} \leqq 1 . \quad$ Q. E. D.

Lemma 4.2. For any $r \geqq 0$ there exists a constant $A>0$ such that for $i+j=k$ with $k=0, \cdots, m-1$

$$
\left\|\sigma(x)^{i \mu} \Lambda^{r+i} D_{t}^{j} v\right\| \leqq c A s^{-(m-k)} w_{r}(s+m-k-1, t, R) .
$$

Proof. It follows from Lemma 4.1 that

$$
\left\|\sigma(x)^{i \mu} \Lambda^{r+i} D_{t}^{j+m-k-1} v\right\| \leqq c A s^{-1} w_{r}(s, t, R) .
$$

Hence we get that

$$
\begin{aligned}
\left\|\sigma(x)^{i \mu} \Lambda^{r+i} D_{t}^{\jmath} v\right\| & \leqq \int_{0}^{t} \cdots \int_{0}^{\tau_{2}}\left\|\sigma(x)^{i \mu} \Lambda^{r+i} D_{t}^{\jmath^{+m-k-1}} v\right\| d \tau_{1} \cdots d \tau_{m-k-1} \\
& \leqq c A s^{-1} R^{r} r !{ }^{k} e^{M r t} \int_{0}^{t} \cdots \int_{0}^{\tau_{2}} \tau_{1}^{s} d \tau_{1} \cdots d \tau_{m-k-1} \\
& \leqq c A s^{-(m-k)} w_{r}(s+m-k-1, t, R)
\end{aligned}
$$

\section{$\S 5$. Estimate of $\Lambda^{r} Q_{1} v$}

We begin with the following lemma.

Lemma 5.1. If $\sigma(x) \in \mathscr{B}\left(\boldsymbol{R}^{n}\right)$ and $0 \leqq \nu<\mu$, then

$$
\left\|\sigma(x)^{\nu} u\right\| \leqq\|u\|^{1-\nu / \mu}\left\|\sigma(x)^{\mu} u\right\|^{\nu / \mu} .
$$

Proof. By Hölder's inequality,

$$
\begin{aligned}
\left\|\sigma(x)^{\nu} u\right\|^{2} & =\int\left|\sigma(x)^{\nu} u\right|^{2} d x=\int|u|^{2(1-\nu / \mu)}\left|\sigma(x)^{\mu} u\right|^{2 \nu / \mu} d x \\
& \leqq\left(\int|u|^{2} d x\right)^{1-\nu / \mu}\left(\int\left|\sigma(x)^{\mu} u\right|^{2} d x\right)^{\nu / \mu} \\
& =\|u\|^{2(1-\nu / \mu)}\left\|\sigma(x)^{\mu} u\right\|^{2 \nu / \mu} .
\end{aligned}
$$

Lemma 5.2. Let $\rho(\alpha, j)=m-j-\nu_{\alpha, j} / \mu$, then

$$
\begin{aligned}
& \sum_{k=1}^{m-1} \sum_{\substack{|\alpha|+>=k \\
|\alpha| \neq 0}}\left\|\sigma(x)^{\nu \alpha, j} \Lambda^{r+|\alpha|} D_{t}^{j} v\right\| \\
& \leqq \sum_{k=1}^{m-1} \sum_{\substack{\alpha++>=k \\
|a| \neq 0}} c A s^{-\rho(\alpha, j)}\{(r+|\alpha|) \cdots(r+1)\}^{-\nu \alpha, j^{k / \mu}} \\
& \times w_{r+|a|}(s+\rho(\alpha, j)-1, t, R) .
\end{aligned}
$$

Proof. From Lemma 4.2 and Lemma 5.1, we obtain that for $|\alpha|+j=k$ and $|\alpha| \neq 0$

$$
\begin{aligned}
& \left\|\sigma(x)^{\nu \alpha, j} \Lambda^{r+|a|} D_{t}^{j} v\right\| \\
& \quad \leqq\left\|\Lambda^{r+|a|} D_{t}^{j} v\right\|^{1-\nu a, j /|a| \mu}\left\|\sigma(x)^{|a| \mu} \Lambda^{r+|a|} D_{t}^{j} v\right\|^{\nu \alpha, j /|a| \mu}
\end{aligned}
$$




$$
\begin{aligned}
= & \left\{c A s^{-(m-j)} w_{r+|\alpha|}(s+m-j-1, t, R)\right\}^{1-\nu \alpha, j /|\alpha| \mu} \\
& \times\left\{c A s^{-(m-k)} w_{r}(s+m-k-1, t, R)\right\}^{\nu \alpha j /|\alpha| \mu} \\
= & c A s^{-\rho(\alpha, j)} R^{r+|\alpha|(1-\nu \alpha, j /|a| \mu)}(r+|\alpha|) !^{\kappa}\{(r+|\alpha|) \cdots(r+1)\}^{-\kappa \nu \alpha, j /|\alpha| \mu} \\
& \times t^{s+\rho(\alpha, j)-1} e^{M(r+|a|(1-\nu \alpha, j /|\alpha| \mu)\} t} \\
\leqq & c A s^{-\rho(\alpha, j)}\{(r+|\alpha|) \cdots(r+1)\}^{-\kappa \nu \alpha, j /|\alpha| \mu} w_{r+|\alpha|}(s+\rho(\alpha, j)-1, t, R) .
\end{aligned}
$$

Q.E.D.

We note that

(5.3) $\nu_{a, j}=0$ or there exists a non-negative integer $p_{a, j}$ such that $p_{\alpha, j} \mu<\nu_{\alpha, j} \leqq\left(p_{\alpha, j}+1\right) \mu$.

Lemma 5.3. For any $r \geqq 0$, the following estimate holds.

$$
\left\|\Lambda^{r} Q_{1} v\right\| \leqq \tilde{c} c A \sum_{k=1}^{m-1} \sum_{\substack{|\alpha+|>=k \\|\alpha| \neq 0}} K_{j}^{a}(s, r) w_{r+|a|}(s+\rho(\alpha, j)-1, t, R),
$$

where

$$
\begin{aligned}
K_{\jmath}^{\alpha}(s, r)= & s^{-\rho(a, j)}\{(r+|\alpha|) \cdots(r+1)\}^{-\kappa \nu} \alpha, \jmath|| a \mid l l \\
& +\sum_{i=1}^{p_{\alpha, j+1}} s^{-\left(m-\jmath-p_{\alpha, \jmath}+\imath-1\right)}\{(r+|\alpha|) \cdots(r+|\alpha|-i+1)\}^{1-\kappa} \\
& \times\left\{(r+|\alpha|-i) \cdots\left(r+|\alpha|-p_{a, \jmath}\right)\right\}^{-\kappa} .
\end{aligned}
$$

Proof.

$$
\begin{aligned}
& \left\|\Lambda^{r} Q_{1} \imath\right\| \leqq \sum_{k=1}^{m-1} \sum_{\substack{|\alpha|+>=k \\
|a| \neq 0}}\left\|\Lambda^{r}\left(\sigma(x)^{\nu} a, \nu a_{a, j}\left(x, t, D_{x}\right) D_{t}^{\jmath} v\right)\right\| \\
& \leqq \sum_{k=1}^{m-1} \sum_{\substack{|\alpha|+j=k \\
|\alpha| \neq 0}}\left\{\left\|\sigma(x)^{2} \alpha, j a_{a, j} \Lambda^{r} D_{t}^{\jmath} v\right\|+\left\|\left[\Lambda^{r}, \sigma(x)^{\nu} \alpha, j a_{a, j}\right] D_{t}^{\jmath} v\right\|\right\} \\
& =I_{1}+I_{2} \text {. }
\end{aligned}
$$

Since

$$
I_{1} \leqq c_{18} \sum_{k=1}^{m-1} \sum_{\substack{|\alpha|+j=k \\|\alpha| \neq 0}}\left\{\left\|\sigma(x)^{\nu} \alpha, j \Lambda^{r+|a|} D_{t}^{j} v\right\|+\left\|\Lambda^{r+\mid a_{1}-1} D_{t}^{j} v\right\|\right\}
$$

the first term has been estimated by Lemma 5.2 and the second term will be estimated within $I_{2}$.

The estimate of $I_{2}$ is similar to the proof of Lemma 4.1. In fact since

$$
\sigma\left(\left[\Lambda^{r}, \sigma(x)^{\nu} \alpha, j a_{\alpha, j}\right]\right)=\sum_{i=1}^{r+|a|-1} \sum_{|\beta|=i} \frac{1}{\beta !} \partial_{\xi}^{\beta}\langle\xi\rangle^{r} D_{x}^{\beta}\left\{\sigma(x)^{\nu} \alpha, j a_{a, j}\right\}+r(x, t, \xi),
$$

if we note that $\nu_{a, j}-|\beta|=\left(p_{\alpha, j}+1-|\beta|\right) \mu+\left(\nu_{a, j}-p_{a, j} \mu-1\right)+(|\beta|-1)(\mu-1)$ and use Lemma A.3 and Lemma 4.2, then we have 


$$
\begin{aligned}
I_{2} \leqq & c_{19} \sum_{k=1}^{m-1} \sum_{\substack{|\alpha|+\gamma=k \\
|\alpha| \neq 0}} \sum_{i=1}^{p_{a, j+1}} c A s^{-\left(m-j-p_{\alpha, j}+i-1\right)} \\
& \times\{(r+|\alpha|) \cdots(r+|\alpha|-i+1)\}^{1-\kappa}\left\{(r+|\alpha|-i) \cdots\left(r+|\alpha|-p_{\alpha, j}\right)\right\}^{-\kappa} \\
& \times w_{r+|\alpha|}\left(s+m-j-p_{a, j}+i-2, t, R\right) .
\end{aligned}
$$

And if we note that $m-j-p_{\alpha, j}+i-1 \geqq \rho(\alpha, j)$, then we can get (5.4).

Q.E.D.

\section{§6. Proof of Theorem 2.1}

In order to prove Theorem 2.1, we prepare several lemmas.

Lemma 6.1. For any $u^{i}(x) \in \Gamma^{(\kappa)}(0 \leqq i \leqq m-1)$ and any $g(x, t) \in \mathcal{B}\left([0, T], \Gamma^{(\kappa)}\right)$, there exists a unique solution $u(x, t) \in \mathcal{B}\left([0, T], \Gamma^{(\kappa)}\right)$ of the equation:

$$
\left\{\begin{array}{l}
Q_{0}\left(x, t, D_{x}, D_{t}\right) u(x, t)=g(x, t) \\
\left.D_{t}^{i} u(x, t)\right|_{t=0}=u^{i}(x), \quad 0 \leqq i \leqq m-1 .
\end{array}\right.
$$

And especially, if $u^{i}(x) \equiv 0(0 \leqq i \leqq m-1)$ and $\left.D_{t}^{i} g(x, t)\right|_{t=0}=0(0 \leqq i \leqq s-1)$, then we obtain that $\left.D_{t}^{i} u(x, t)\right|_{t=0}=0(0 \leqq i \leqq s+m-1)$, where $s$ is a positive integer.

Proof. It follows from Proposition 2.1 that there exists a unique solution $u(x, t) \in \mathscr{B}\left([0, T], H^{\infty}\right)$ of (6.1). Therefore let us show that $u(x, t) \in \mathscr{B}([0, T]$, $\left.\Gamma^{(\kappa)}\right)$.

For any fixed integer $s \geqq 1$, let $u_{s}(x, t)$ be

$$
u_{s}(x, t)=u(x, t)-\left.\sum_{j=0}^{s+m-1} \frac{t^{j}}{j !} \partial^{j} u(x, t)\right|_{t=0},
$$

then $u_{s}(x, t)$ satisfies the equation

$$
\begin{aligned}
Q_{0} u_{s}(x, t) & =g(x, t)-Q_{0}\left(\left.\sum_{j=0}^{s+m-1} \frac{t^{\jmath}}{j !} \partial^{j} u(x, t)\right|_{t=0}\right) \\
& \equiv g_{s}(x, t) .
\end{aligned}
$$

Therefore we get that $g_{s}(x, t) \in \mathscr{B}\left([0, T], \Gamma^{(\kappa)}\right)$ such that $\left.D_{t}^{i} g_{s}(x, t)\right|_{t=0}=0,0 \leqq i$ $\leqq s-1$. From the consequence of $\S 4$, it is easily seen that $u_{s}(x, t) \in \mathscr{B}([0, T]$, $\left.\Gamma^{(\kappa)}\right)$. Hence we obtain that $u(x, t) \in \mathscr{B}\left([0, T], \Gamma^{(\kappa)}\right)$.

As to the latter, since $D_{t}^{m} u=\left(D_{t}^{m} u-Q_{0} u\right)+g$, we can get that $\left.D_{t}^{m} u\right|_{t=0}=0$. And since $D_{t}^{m+1} u=D_{t}\left(D_{t}^{m} u-Q_{0} u\right)+D_{t} g$, we get that $\left.D_{t}^{m+1} u\right|_{t=0}=0$. Hence if we repeat these steps, we have that $\left.D_{t}^{i} u\right|_{t=0}=0,0 \leqq i \leqq s+m-1$.

Q.E.D.

Lemma 6.2. Let $u_{j}(x, t)$ be the solution of $(2.6)_{j}$, then $u_{j}(x, t) \in \mathscr{B}([0, T]$, $\left.\Gamma^{(\kappa)}\right)$ for $j \geqq 0$. Moreover for $j \geqq 1,\left.D_{t}^{i} u_{j}(x, t)\right|_{t=0}=0,0 \leqq i \leqq m+2 j-3$.

Proof. It follows from the first assersion of Lemma 6.1 that $u_{0}(x, t) \in$ 
$\mathcal{B}\left([0, T], \Gamma^{(\kappa)}\right)$. Since $Q_{1} u_{0} \in \mathcal{B}\left([0, T], \Gamma^{(\kappa)}\right)$, if we use Lemma 6.1 once more, we can get that $u_{1}(x, t) \in \mathscr{B}\left([0, T], \Gamma^{(\kappa)}\right)$. 'Therefore repeating these steps we have $u_{\jmath}(x, t) \in \mathscr{B}\left([0, T], \Gamma^{(\kappa)}\right)$ for $j \geqq 0$.

Next from the second assersion of Lemma 6.1 and the form of $(2.6)_{1}$, $\left.D_{t}^{i} u_{1}\right|_{t=0}=0,0 \leqq i \leqq m-1$. Since $\left.D_{t}^{i} Q_{1} u_{1}\right|_{t=0}=0$ for $i=0,1$, we obtain that $\left.D_{t}^{i} u_{2}\right|_{t=0}$ $=0$ for $0 \leqq i \leqq m+1$. Similarly, we conclude the second assersion of Lemma 6.2.

Q.E.D.

From Lemma 6.2, for any fixed integer $s \geqq 1$, there exists $N=N^{\top}(s) \in N$ such that for any $j \geqq N-1,\left.D_{l}^{i} u_{j}(x, t)\right|_{\iota=0}=0,0 \leqq i \leqq s-1$. Therefore we may assume that for any $r \geqq 0$

$$
\left\|\Lambda^{r} Q_{1} u_{N-1}\right\| \leqq c w_{r}(s, t, R),
$$

where $c$ and $R$ are positive constants.

Lemma 6.3. Under (6.2), if $1 \leqq \kappa<\rho /(\rho-1)$, there exist constants $\tilde{A}, B, \gamma>0$ which are independent of $r$ such that

$$
\left\|\Lambda^{r} u_{N+n}\right\| \leqq c \tilde{A} B^{n} n^{-r n} w_{r}\left(s, t, 2^{n} R\right)
$$

for $n=0,1,2, \cdots$.

Proof. From (6.2) and Lemma 4.2, we get that

$$
\left\|\sigma(x)^{\left|\alpha_{1}\right| \mu} \Lambda^{r+\left|a_{1}\right|} D_{t}^{j_{1}} u_{N}\right\| \leqq c A s^{-\left(m-j-\left|a_{1}\right|\right)} T^{m-1} w_{r}(s, t, R) .
$$

It follows from Lemma 5.3 that

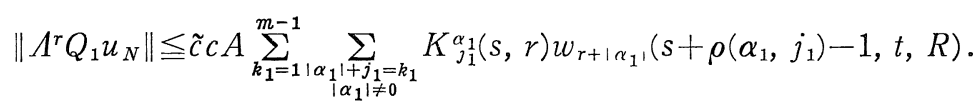

If we use Lemma 4.2 , we have that

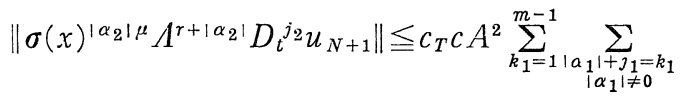

$$
\begin{aligned}
& \left(s+\rho\left(\alpha_{1}, j_{1}\right)-1\right)^{-\left(m-j_{2}-\left|a_{2}\right|\right)} K_{j_{1}}^{\alpha_{1}}(s, r) w_{r+\left|\alpha_{1}\right|}\left(s+\rho\left(\alpha_{1}, j_{1}\right)-1, t, R\right),
\end{aligned}
$$

where $c_{T}=\tilde{c} T^{m-1}$.

Applying Lemma 5.3 again, we obtain, that

$$
\begin{aligned}
& \left\|A^{r} Q_{1} u_{N+1}\right\| \leqq \tilde{c} c_{T} c A^{2} \sum_{k_{1}, k_{2}=1}^{m-1} \sum_{\substack{\left|\alpha_{1}+j_{1}=k_{1}\right| \alpha_{2}\left|+\alpha_{1}=\alpha_{2}=k_{2}\\
\right| \alpha_{2} \mid \neq 0}} \\
& \quad K_{J_{1}}^{\alpha_{1}}(s, r) K_{J_{2}}^{\alpha_{2}}\left(s+\rho\left(\alpha_{1}, j_{1}\right)-1, r+\left|\alpha_{1}\right|\right) \\
& \quad \times w_{r+\left|\alpha_{1}\right|+\left|\alpha_{2}\right|}\left(s+\rho\left(\alpha_{1}, j_{1}\right)-1+\rho\left(\alpha_{2}, j_{2}\right)-1, t, R\right)
\end{aligned}
$$

From Lemma 4.2 , we get that 


$$
\begin{aligned}
& \left\|\sigma(x)^{\left|\alpha_{3}\right| \mu} \Lambda^{r+\left|\alpha_{3}\right|} D_{t}^{j_{3}} u_{N+2}\right\| \leqq c A\left(c_{T} A\right)^{2} \sum \sum \sum K_{j_{1}}^{\alpha_{1}}(s, r) K_{j_{1}, j_{2}}^{\alpha_{1}, \alpha_{2}}(s, r) \\
& \quad \times\left(s+\rho\left(\alpha_{1}, j_{1}\right)-1+\rho\left(\alpha_{2}, j_{2}\right)-1\right)^{-\left(m-j_{3}-\left|\alpha_{3}\right|\right)} \\
& \quad \times w_{r+\left|\alpha_{1}\right|+\left|\alpha_{2}\right|}\left(s+\rho\left(\alpha_{1}, j_{1}\right)-1+\rho\left(\alpha_{2}, j_{2}\right)-1, t, R\right),
\end{aligned}
$$

where $K_{j_{1}, j_{2}}^{\alpha_{1}, \alpha_{2}}(s, r)=K_{j_{2}}^{\alpha_{2}}\left(s+\rho\left(\alpha_{1}, j_{1}\right)-1, r+\left|\alpha_{1}\right|\right)$.

We set

$$
\begin{aligned}
& K_{j_{1}, \cdots ; j_{l}}^{\alpha_{1}, \cdots, \alpha},(s, r) \\
& \quad=K_{J_{l}}^{\alpha} l\left(s+\rho\left(\alpha_{1}, j_{1}\right)-1+\cdots+\rho\left(\alpha_{l-1}, j_{l-1}\right)-1, r+\left|\alpha_{1}\right|+\cdots+\left|\alpha_{l-1}\right|\right) .
\end{aligned}
$$

Inductively we obtain that for any $n \geqq 0$

$$
\begin{aligned}
& \left\|A^{r} u_{N+n}\right\| \leqq c A\left(c_{T} A\right)^{n} \sum \cdots \sum K_{j_{1}}^{\alpha_{1}} \cdots K_{j_{1}, \cdots, j_{n}}^{\alpha_{1}, \cdots, a_{n}} \\
& \quad \times w_{r+\left|\alpha_{1}\right|+\cdots+\left|\alpha_{n}\right|}\left(s+\rho\left(\alpha_{1}, j_{1}\right)-1+\cdots+\rho\left(\alpha_{n}, j_{n}\right)-1, t, R\right) .
\end{aligned}
$$

By the way,

$$
\begin{aligned}
& K_{j_{1}}^{\alpha_{1}} \cdots K_{j_{1}, \cdots, j_{n}}^{\alpha_{1}, \cdots, \alpha_{n}} \\
& =\Sigma \cdots \sum s^{-a_{1}}(r+1)^{-b_{1}^{1}} \cdots\left(r+\left|\alpha_{1}\right|\right)^{-b_{1}^{1 \alpha_{1} i}}
\end{aligned}
$$

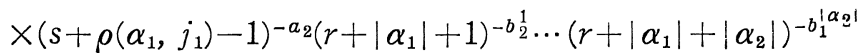

$$
\begin{aligned}
& \times \cdots \times\left(s+\rho\left(\alpha_{1}, j_{1}\right)-1+\cdots+\rho\left(\alpha_{n-1}, j_{n-1}\right)-1\right)^{-a_{n}} \\
& \times\left(r+\left|\alpha_{1}\right|+\cdots+\left|\alpha_{n-1}\right|+1\right)^{-b_{n}^{1}} \cdots\left(r+\left|\alpha_{1}\right|+\cdots+\left|\alpha_{n}\right|\right)^{-b_{n}^{\mid \alpha} n^{1}},
\end{aligned}
$$

where $a_{k} \in\left\{\rho\left(\alpha_{k}, j_{k}\right), m-j_{k}-p_{a_{k}, j_{k}}+i_{k}-1\right\}$ and $b_{k}^{l} \in\left\{\kappa \nu_{a_{k}, j_{k}} /\left|\alpha_{k}\right| \mu, \kappa-1, \kappa, 0\right\}$. We note the following.

(6.4) If $a_{k}=\rho\left(\alpha_{k}, j_{k}\right)$, then $b_{k}^{1}, \cdots, b_{k}^{\left|\alpha_{k}\right|}=\kappa \nu_{\alpha_{k}, j_{k}} /\left|\alpha_{k}\right| \mu$.

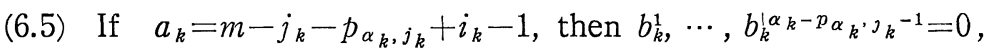

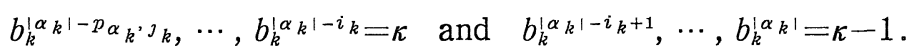

Let $s \geqq \max \{\rho(\alpha, j)-1\}, \omega=\min \{\rho(\alpha, j)-1\}$ and $a=\min \left\{a_{k}\right\}$ and if we use Lemma A.4, then we have

$$
\begin{aligned}
s^{-a_{1}} & \cdots\left(s+\rho\left(\alpha_{1}, j_{1}\right)-1+\cdots+\rho\left(\alpha_{n-1}, j_{n-1}\right)-1\right)^{-a_{n}} \\
& \leqq\left(\rho\left(\alpha_{n}, j_{n}\right)-1\right)^{-a_{1}} \cdots\left(\rho\left(\alpha_{1}, j_{1}\right)-1+\cdots+\rho\left(\alpha_{n}, j_{n}\right)-1\right)^{-a_{n}} \\
& \leqq \omega^{-\left(a_{1}+\cdots+a_{n}\right)} 1^{-a_{1}} \cdots n^{-a_{n}} \\
& \leqq \omega^{-a n} A_{1} R_{\perp}^{n} n^{-\left(a_{1}+\cdots+a_{n}\right)} .
\end{aligned}
$$

Let $r=0$, them by Lemma A.4 again,

$$
\begin{aligned}
& (r+1)^{-b_{1}^{1}} \cdots\left(r+\left|\alpha_{1}\right|\right)^{-b_{1}^{\left|\alpha_{1}\right|}} \\
& \quad \times \cdots \times\left(r+\left|\alpha_{1}\right|+\cdots+\left|\alpha_{n-1}\right|+1\right)^{-b_{n}^{1}} \cdots\left(r+\left|\alpha_{1}\right|+\cdots+\left|\alpha_{n}\right|\right)^{-b_{n}^{\mid \alpha} \mid}
\end{aligned}
$$




$$
\begin{aligned}
& \leqq A_{1} R_{1}^{n}\left(\left|\alpha_{1}\right|+\cdots+\left|\alpha_{n}\right|\right)^{-\left(b_{1}^{1}+\cdots+b_{n}^{\mid \alpha} n^{\prime}\right)} \\
& \leqq A_{1} R_{1}^{n} n^{-\left(b_{1}^{1}+\cdots+b_{n}^{\mid \alpha} n^{\prime}\right)} .
\end{aligned}
$$

Further we estimate

$$
\begin{aligned}
& w_{r+\left|a_{1}\right|+\cdots+\left|\alpha_{n}\right|}\left(s+\rho\left(\alpha_{1}, j_{1}\right)-1+\cdots+\rho\left(\alpha_{n}, j_{n}\right)-1, t, R\right) . \\
& R^{r+\left|a_{1}\right|+\cdots+\left|a_{n}\right|} \leqq R^{r} R^{(m-1) n}, \\
& t^{s+\rho\left(a_{1}, j_{1}\right)-1+\cdots+\rho\left(a_{n}, j_{n}\right)-1} \leqq t^{s} T^{\varpi n}, \text { where } \tilde{\omega}=\max \{\rho(\alpha, j)-1\}, \\
& e^{M\left(r+\left|a_{1}\right|+\cdots+\left|\alpha_{n}\right|\right) t} \leqq e^{M r t} e^{M(m-1) T n} .
\end{aligned}
$$

Using Lemma A.5,

$$
\begin{aligned}
& \left(r+\left|\alpha_{1}\right|+\cdots+\left|\alpha_{n}\right|\right) !^{\kappa} \leqq 2^{\left(r+\left|a_{1}\right|+\cdots+\left|a_{n}\right|\right) \kappa} r !^{\kappa}\left(\left|\alpha_{1}\right|+\cdots+\left|\alpha_{n}\right|\right) !^{\kappa} \\
& \quad \leqq 2^{r \kappa} 2^{(m-1) n \kappa} r !^{\kappa} A_{2} R_{2}^{n} n^{\left(\left|a_{1}\right|+\cdots+\left|a_{n}\right|\right) \kappa} .
\end{aligned}
$$

Therefore we obtain that

$$
\begin{aligned}
& \left\|\Lambda^{r} u_{N+n}\right\| \\
& \leqq c A A_{1}^{2} A_{2}\left(c_{T} A R_{1}^{2} R_{2} \omega^{-a} R^{m-1} 2^{(m-1) \kappa} T^{\varpi} e^{M I(m-1) T}\right)^{n} w_{r}\left(s, t, 2^{\kappa} R\right) \\
& \times \sum \cdots \sum n^{\left(\left|a_{1}\right|+\cdots+\left|a_{n}\right|\right) \kappa-\left(a_{1}+\cdots+a_{n}\right)-\left(b_{1}^{1}+\cdots+b_{n}^{\mid \alpha} n^{\mid}\right)} .
\end{aligned}
$$

Let $q$ be the number of $\rho\left(\alpha_{k}, j_{k}\right)$ 's in $\left\{a_{k}\right\}_{1 \leqq k \leqq n}$ and if we remember (6.4), (6.5) and (5.3), then we obtain that

$$
\begin{aligned}
\left(a_{1}+\cdots\right. & \left.+a_{n}\right)+\left(b_{1}^{1}+\cdots+b_{n}^{\left|\alpha_{n}\right|}\right)-\left(\left|\alpha_{1}\right|+\cdots+\left|\alpha_{n}\right|\right) \kappa \\
= & \rho\left(\alpha_{1}, j_{1}\right)+\cdots+\rho\left(\alpha_{q}, j_{q}\right) \\
& +\left(m-j_{q+1}-p_{a_{q+1}, j_{q+1}}+i_{q+1}-1\right)+\cdots+\left(m-j_{n}-p_{a_{n} \jmath_{n}}+i_{n}-1\right) \\
& +\kappa \nu_{a_{1}, j_{1}} / \mu+\cdots+\kappa \nu_{a_{q}, \jmath_{q}} / \mu \\
& +\kappa\left(p_{a_{q+1}, j_{q+1}}+1-i_{q+1}\right)+\cdots+\kappa\left(p_{a_{n}, \jmath_{n}}+1-i_{n}\right) \\
& +(\kappa-1) i_{q+1}+\cdots+(\kappa-1) i_{n}-\left(\left|\alpha_{1}\right|+\cdots+\left|\alpha_{n}\right|\right) \kappa \\
= & \left(\rho\left(\alpha_{1}, j_{1}\right)+\kappa \nu_{a_{1}, j_{1}} / \mu-\left|\alpha_{1}\right| \kappa\right)+\cdots+\left(\rho\left(\alpha_{q}, j_{q}\right)+\kappa \nu_{a_{q}, \jmath_{q}} / \mu-\left|\alpha_{q}\right| \kappa\right) \\
& +\left(m-j_{q+1}-p_{a_{q+1}, j_{q+1}}-1+p_{a_{q+1}} j_{q+1} \kappa+\kappa-\left|\alpha_{q+1}\right| \kappa\right) \\
& +\cdots+\left(m-j_{n}-p_{a_{n}, j_{n}}-1+p_{\alpha_{n}, j_{n}} \kappa+\kappa-\left|\alpha_{n}\right| \kappa\right) \\
\geqq & \left(m-j_{1}-\nu_{a_{1}, j_{1}} / \mu+\kappa \nu_{a_{1}, j_{1}} / \mu-\left|\alpha_{1}\right| \kappa\right) \\
& +\cdots+\left(m-j_{n}-\nu_{a_{n}, j_{n}} / \mu+\kappa \nu_{a_{n}, j_{n}} / \mu-\left|\alpha_{n}\right| \kappa\right) \\
= & \left(m-j_{1}-\left|\alpha_{1}\right|\right)\left\{\left(m-j_{1}-\nu_{a_{1}} \jmath_{1} / \mu\right) /\left(m-j_{1}-\left|\alpha_{1}\right|\right.\right.
\end{aligned}
$$




$$
\begin{aligned}
& \left.-\left(\left(m-j_{1}-\nu_{\alpha_{1}, j_{1}} / \mu\right) /\left(m-j_{1}-\left|\alpha_{1}\right|\right)-1\right) \kappa\right\}+\cdots \\
& +\left(m-j_{n}-\left|\alpha_{n}\right|\right)\left\{\left(m-j_{n}-\nu_{a_{n}, j_{n}} / \mu\right) /\left(m-j_{n}-\left|\alpha_{n}\right|\right)\right. \\
& \left.-\left(\left(m-j_{n}-\nu_{\alpha_{n}, j_{n}} / \mu\right) /\left(m-j_{n}-\left|\alpha_{n}\right|\right)-1\right) \kappa\right\} \\
\geqq & n\{\rho-(\rho-1) \kappa\} .
\end{aligned}
$$

This completes the proof.

Q.E.D.

Corollary 6.1. If $1 \leqq \kappa<\rho /(\rho-1)$, the formal solution

$$
u(x, t)=\sum_{j=0}^{\infty} u_{\jmath}(x, t)
$$

converges in $\mathcal{B}\left([0, T], \Gamma^{(\kappa)}\right)$.

Proof. If we devide $u(x, t)$ as

$$
u(x, t)=\sum_{j=0}^{N-1} u_{\jmath}(x, t)+\sum_{j=N}^{\infty} u_{\jmath}(x, t),
$$

then this Corollary immidiately follows from Lemma 6.2 and Lemma 6.3.

Q.E.D.

Hence we obtain the existence of solutions.

Next we shall show the uniqueness of solutions.

Lemma 6.4. If $u(x, t) \in \mathscr{B}\left([0, T], \Gamma^{(\kappa)}\right)$ is a solution of the Cauchy problem:

$$
\left\{\begin{array}{l}
P\left(x, t, D_{x}, D_{t}\right) u(x, t)=0 \\
\left.D_{t}^{i} u(x, t)\right|_{t=0}=0, \quad 0 \leqq i \leqq m-1,
\end{array}\right.
$$

where $1 \leqq \kappa<\rho /(\rho-1)$, then $u(x, t) \equiv 0$.

Proof. We may assume that for sufficiently large $s$ there exist constants $c, R>0$ such that

$$
\left\|\Lambda^{r} u\right\| \leqq c w_{r}(s, t, R) \quad \text { for any } r \geqq 0 .
$$

Therefore similar to the proof of Lemma 6.3, we can obtain that

$$
\left\|\Lambda^{r} u\right\| \leqq c \tilde{A} B^{n} n^{-r n} w_{r}(s, t, \tilde{R}) .
$$

Let $n$ be infinity, then we have that $u(x, t) \equiv 0$.

Q.E.D.

Acknowledgement: The author would like to thank Prof. Shoji Irie and Prof. Hitoshi Uryu for their useful advices.

\section{Appendix}

Following Igari [2] and Uryu [12], we introduce a certain class of pseudo- 
differential operators.

Definition A.1. 1) For any $m \in R$ and $\kappa>1$, we denote by $S^{m}(\kappa)$ the set of functions $h(x, \xi) \in C^{\infty}\left(\boldsymbol{R}^{n} \times \boldsymbol{R}^{n}\right)$ satisfying the property that for any $\alpha$, $\beta$, there exist constants $c_{\alpha}$ and $R$ such that

$$
\left|\partial_{\xi}^{\alpha} D_{x}^{\beta} h(x, \xi) i \leqq c_{\alpha} R^{|\beta|}\right| \beta \mid !^{\kappa}\langle\xi\rangle^{m-|a|} \quad \text { for } \quad(x, \xi) \in \mathbb{R}^{n} \times \mathbb{R}^{n} \text {. }
$$

2) For any $h(x, \xi) \in S^{m}(\kappa)$, we shall define a semi-norm of $h(x, \xi)$ such that for any integer $l \geqq 0$

$$
|h(x, \xi)|_{l}=\max _{|a+\beta| \leqq l} \sup _{(x, \xi) \in R^{n} \times R^{n}}\left|\partial_{\xi}^{\alpha} D_{x}^{\beta} h(x, \xi)\right|\langle\xi\rangle^{-m+|a|} .
$$

Now we can define a pseudo-differential operator with a symbol $h(x, \xi) \in$ $S^{m}(\kappa)$ as follows.

$$
H\left(x, D_{x}\right) u(x)=(2 \pi)^{-n} \int e^{2 x \cdot \xi} h(x, \xi) \hat{u}(\xi) d \xi .
$$

Lemma A.1 (see Igari [2]). Let $h(x, \xi) \in S^{m}(\kappa)$ and $r$ bo non-negative irtegers. Then

$$
\sigma\left(\Lambda^{r} H\right)=\sum_{j=1}^{N-1} \sum_{|\alpha|=j} \frac{1}{\alpha !} \partial_{\xi}^{\alpha}\langle\xi\rangle^{r} D_{x}^{a} h(x, \xi)+r_{N}(x, \xi),
$$

where $N=r+m$. And for any integer $l \geqq 0$, there exist constants $c_{l}, R>0$ such that

and

$$
\left|D_{x}^{\alpha} h(x, \xi)\langle\xi\rangle^{-m}\right|_{l} \leqq c_{l} R^{|a|-m}(|\alpha|-m) !^{k}
$$

$$
\left|r_{N}(x, \xi)\right|_{l} \leqq c_{l} R^{r} r !^{\kappa} \text {. }
$$

The following lemma is well-known.

Lemma A.2. For any $h(x, \xi) \in S^{0}$, there exist a constant $c$ and non-negative integer $l$ dependent only on dimension $n$ such that

$$
\left\|H\left(x, D_{x}\right) u\right\| \leqq c|h(x, \xi)|_{\imath}\|u\| .
$$

Lemma A.3 (see Uryu [12]). Under the assumptions of Lemma A.1, if we denote $h_{\jmath}(x, \xi)$ by

$$
h_{\jmath}(x, \xi)=\sum_{|\alpha|=j} \frac{1}{\alpha !} \partial_{\xi}^{a}\langle\xi\rangle^{r} D_{x}^{a} h(x, \xi),
$$

then there exist $\hat{c}, \hat{R}>0$ such that

$$
\begin{aligned}
& \left\|H_{\jmath}\left(x, D_{x}\right) u\right\| \leqq \hat{c} \hat{R}^{j-m}(j-m) !^{\kappa}\left(\begin{array}{c}
r \\
j
\end{array}\right)\left\|\Lambda^{m+r-\jmath} u\right\| \quad \text { for } 1 \leqq j \leqq r, \\
& \left\|H_{\jmath}\left(x, D_{x}\right) u\right\| \leqq \hat{c} \hat{R}^{j-m}(j-m) !^{\kappa}\left\|\Lambda^{m+r-j} u\right\| \quad \text { for } \quad r+1 \leqq j \leqq N-1, \\
& \left\|R_{N}\left(x, D_{x}\right) u\right\| \leqq \hat{c} \hat{R}^{r} r !^{\kappa}\|u\| .
\end{aligned}
$$


Lemma A.4. Let $\left\{i_{1}, \cdots, i_{n}\right\}$ be a subset of $\left\{a_{1}, \cdots, a_{m}\right\}$, then there exist constants $A_{1}, R_{1}>0$ such that

$$
n^{i_{1}+\cdots+i_{n}} \leqq A_{1} R_{1}^{n} 1^{i_{1}} 2^{i_{2}} \cdots n^{i_{n}} .
$$

Proof. Set $S=n^{i_{1}+\cdots+i_{n}} / 1^{i_{1}} \cdots n^{i_{n}}$. Then

$$
\begin{aligned}
S & =(n / 1)^{i_{1}}(n / 2)^{i_{2}} \cdots(n / n)^{i_{n}} \\
& \leqq(n / 1)^{a}(n / 2)^{a} \cdots(n / n)^{a} \\
& =\left(n^{n} / n !\right)^{a}, \quad \text { where } a=\max \left\{a_{1}, \cdots, a_{m}\right\} .
\end{aligned}
$$

Using Stirling's formula, we can get the desired inequality.

Q.E.D.

Lemma A.5. Let $\left\{i_{1}, \cdots, i_{n}\right\} \subset\{1, \cdots, m-1\}$, then there exist constants $A_{2}$, $R_{2}>0$ such that

$$
\left(i_{1}+\cdots+i_{n}\right) ! \leqq A_{2} R_{2}^{n} n^{i_{1}+\cdots+i_{n}} .
$$

Proof. By Stirling's formula, we obtain that

$$
\begin{aligned}
\left(i_{1}+\cdots+i_{n}\right) & ! A_{2}\left(i_{1}+\cdots+i_{n}\right)^{\left(i_{1}+\cdots+i_{n}\right)} \\
& \leqq A_{2}\{n(m-1)\}^{\left(i_{1}+\cdots+i_{n}\right)} \\
& \leqq A_{2}(m-1)^{n(m-1)} n^{i_{1}+\cdots+i_{n}} .
\end{aligned}
$$

\section{References}

[1] Bronstein, M.D., The Cauchy problem for hyperbolic operators with characteristics of variable multiplicity, Trans. Moscow Math. Soc., 41 (1982), 87-103.

[2] Igari, K., An admissible data class of the Cauchy problem for non-strictly hyperbolic operators, J. Math. Kyoto Univ., 21 (1981), 351-373.

[3] Ivrii, V. Ja., Correctness of the Cauchy problem in Gevrey classes for nonstrictly hyperbolic operators, Math. USSR Sb., 25 (1975), 365-387.

[4] - Cauchy problem conditions for hyperbolic operators with characteristics of variable multiplicity for Gevrey classes, Siberian Math. J., 17 (1976), 921-931.

[5] Kajitani, K., Cauchy problem for nonstrictly hyperbolic systems in Gevrey classes, J. Math. Kyoto Univ., 23 (1983), 599-616.

[6] Leray J. and Ohya, Y. Equations et Systémes Non-Linéaies Hyperboliques NonStricts, Math. Ann., 170 (1967), 167-205.

[7] Nishitani, T., Energy inequality for non strictly hyperbolic operators in the Gevrey class, J. Math. Kyoto Univ., 23 (1983), 739-773.

[8] Ohya, Y., Le problème de Cauchy pour les équations hyperboliques à caractéristique multiple, J. Math. Soc. Japan, 16 (1964), 268-286.

[9] Steinberg, S., Existence and Uniqueness of Solutions of Hyperbolic Equations Which are Not Necessarily Strictly Hyperbolic, J. Diff. Eq., 17 (1975), 119-153.

[10] Trepreau, J.M., Le problem de Cauchy hyperbolique dans les classes d'ultrafonctions et d'ultradistributions, Comm. in P.D.E., 4 (1979), 339-387.

[11] Uryu, H., The Cauchy problem for weakly hyperbolic equations (II) ; Infinite degenerate case, Tokyo J. Math., 3 (1980), 99-113. 
[12] - Conditions for well-posedness in Gevrey classes of the Cauchy problems for Fuchsian hyperbolic operators, Publ. RIMS Kyoto Univ., 21 (1985), 355-383.

[13] Uryu, H. and Itoh, S. Well-posedness in Gevrey classes of the Cauchy problems for some second order weakly hyperbolic operators, to appear in Funkcial. Ekvac. 
